\title{
The contribution of walking to work to adult physical activity levels: a cross sectional study
}

Suzanne Audrey ${ }^{1 *}$, Sunita Procter ${ }^{1}$ and Ashley R Cooper ${ }^{2,3}$

\begin{abstract}
Objective: To objectively examine the contribution to adult physical activity levels of walking to work.

Methods: Employees ( $n=103 ; 36.3 \pm 11.7$ years) at 17 workplaces in south-west England, who lived within 2 miles $(3.2 \mathrm{~km})$ of their workplace, wore Actigraph accelerometers for seven days during waking hours and carried GPS receivers during the commute to and from work. Physical activity volume (accelerometer counts per minute (cpm)) and intensity (minutes of moderate to vigorous physical activity (MVPA)) were computed overall and during the walk to work.

Results: Total weekday physical activity was $45 \%$ higher in participants who walked to work compared to those travelling by car (524.6. \pm 170.4 vs $364.6 \pm 138.4 \mathrm{cpm}$ ) and MVPA almost 60\% higher (78.1 \pm 24.9 vs $49.8 \pm 25.2$ minutes per day). No differences were seen in weekend physical activity, and sedentary time did not differ between the groups. Combined accelerometer and GPS data showed that walking to work contributed $47.3 \%$ of total weekday MVPA.

Conclusions: Walking to work was associated with overall higher levels of physical activity in young and middle-aged adults. These data provide preliminary evidence to underpin the need for interventions to increase active commuting, specifically walking, in adults.
\end{abstract}

Keywords: Physical activity measurement, Accelerometer, Walking, Adult physical activity guidelines

\section{Introduction}

There is compelling evidence that regular physical activity is effective in the prevention of chronic diseases (including cardiovascular disease, type 2 diabetes, some cancers, hypertension, obesity, depression and osteoporosis) and premature death, with the greatest improvements in health status seen when people who are least active become physically active [1,2]. In the United Kingdom (UK) it is currently recommended that adults should aim to undertake at least 150 minutes of moderate intensity physical activity in bouts of 10 minutes or more throughout the week $[3,4]$ but many adults in the United Kingdom and other high-income countries do not achieve this [1,4-6]. Increasing physical activity levels, particularly among the most inactive, is an important aim of current public health policy in the UK [1,7-9].

\footnotetext{
* Correspondence: suzanne.audrey@bristolac.uk

${ }^{1}$ School of Social and Community Medicine, University of Bristol, Canynge Hall, Whatley Road, Bristol BS8 2PS, UK

Full list of author information is available at the end of the article
}

\section{The benefits of active travel}

One approach to increasing physical activity levels is to promote active travel i.e. walking and cycling. There is increasing evidence of the link between adult obesity levels and travel behaviour, one indicator of which is that countries with highest levels of active travel generally have the lowest obesity rates [10]. Experts in many World Health Organisation (WHO) countries agree that significant public health benefits can be realised through greater use of active transport modes [11]. For example, a systematic review of trials and cohort studies found modest but consistent support for the positive health effects of active travel, including a suggested positive effect on diabetes [12]. Other studies have shown a protective association between active travel and cardiovascular risk $[13,14]$ and perceived health status [15]. Furthermore, cost benefit analysis for the UK Department for Transport suggests the ratio of benefits to costs were high [16]. The suggested benefits to employers of promoting active travel schemes include: increased productivity, a reduction in sick leave, improved public image as a 
result of lowering the workplace's carbon footprint, and savings in providing car parking facilities [14,17-20].

\section{Walking as active travel}

Walking has been described as near perfect exercise [21]. It is a popular, familiar, convenient and free form of exercise that can be incorporated into everyday life and sustained into older age. It is also a carbon neutral mode of transport that has declined in recent decades in parallel with the growth in car use [1]. Even walking at a moderate pace of three miles/hour (five $\mathrm{km} /$ hour) expends sufficient energy to meet the definition of moderate intensity physical activity [22]. Hence there are compelling reasons to encourage people to walk more, not only to improve their own health but also to address the problems of climate change [23-26].

In the UK, there are substantial opportunities to increase walking by replacing short journeys undertaken by car. For example, the 2011 National Travel Survey showed $22 \%$ of all car trips were shorter than two miles $(3.2 \mathrm{~km})$ in length, while $18 \%$ of trips of less than one mile were made by car [27]. An opportunity for working adults, especially those who live relatively close to their workplace, to accumulate the recommended moderate activity levels may be through the daily commute.

Although cycling is also an important mode of active transportation, walking may be perceived as a cheaper and safer option for those who are currently inactive: it requires no special equipment and is less likely to involve direct competition with motorised traffic for road space. In addition, for longer journeys, walking can more easily be combined with other transport modes such as buses and trains. In their study promoting active travel to work, Mutrie et al. (2002) [28] found the intervention group almost twice as likely to report an increase in walking during their journey to work as the control group at six months (odds ratio of 1.93, 95\% confidence intervals 1.06 to 3.52 ) but there was no increase in cycling.

\section{Measuring active travel}

Active travel has been associated with increased physical activity in studies using self report [29]. However, a systematic review comparing direct versus self-report measures for assessing physical activity in adults found self-report measures were both higher and lower than directly measured levels [30]. This questions the validity and reliability of self-report measures, and also undermines efforts to correct for self-report differences. However, very few studies have objectively measured the contribution of walking, particularly walking to work, to adult physical activity levels [31]. In the US, a crosssectional study included 2,364 participants enrolled in the Coronary Artery Risk Development in Young Adults
(CARDIA) study who worked outside the home during year 20 of the study (2005-2006) [32]. Associations were examined between walking or cycling to work and objective MVPA using accelerometers and active commuting was found to be positively associated with fitness in men and women, and inversely associated with BMI, obesity, triglyceride levels, blood pressure and insulin level in men. The authors concluded that active commuting should be investigated as a means of maintaining or improving health.

Objective measures of physical activity are more common in studies examining children's commute to school. Studies investigating differences in physical activity between children who walk to school and those who travel by car have shown that children who walk to school have substantially higher physical activity than car travellers [33]. More recently, longitudinal studies have shown that a change of travel mode from passive (car/bus) to active (walking) is associated with an increase in overall daily physical activity, whilst physical activity declines if children adopt car travel instead of walking to school $[34,35]$. Spatial segmentation studies have confirmed the importance of walking to school to children's overall physical activity, showing that approximately a third of daily MVPA is acquired in the school journey [36].

\section{The walk to work study}

In the UK, public health guidance on workplace health promotion from the National Institute for Health and Clinical Excellence (NICE) has asserted that, although a range of schemes exist to encourage employees to walk or cycle to work, little is known about their impact and the measures of physical activity used are often based on self-report [37]. In this context, the Walk to Work feasibility study [38] was developed in the south-west of England using objective measurements of physical activity. The main study is examining the feasibility of implementing and evaluating an intervention through which Walk to Work promoters are recruited and trained to encourage fellow employees, who do not currently walk or cycle to work, to increase the amount of walking they undertake during the daily commute. This paper focuses on baseline data to examine the association between travel mode to work and objectively measured physical activity in adults.

\section{Methods}

\section{Recruitment}

Workplaces were contacted by email through the local Chambers of Commerce and by post through a publicly available list of employers in the area. Each workplace was sent an information sheet about the Walk to Work study and asked to return a form to indicate expressions of interest. Following additional information about what the study entailed, a total of 17 workplaces were recruited 
to the study: eight small ( $\leq 50$ employees), five medium (51-250 employees) and four large ( $>250$ employees).

Eligible employees were adults in full or part-time employment who lived within two miles (just over three kilometres) of their workplace and were capable of walking to work regardless of their current mode of transport for commuting. Participating workplaces were asked to identify eligible employees by matching postcodes of workplace and home address and calculating distance using an online calculator (Walkit.com). It was a requirement of the research ethics committee that this was done by workplaces and not the research team. Eligible employees were then given an information sheet and a letter of invitation to take part in the study. Written informed consent was obtained from each participant before data collection commenced.

The study was given ethical approval by the University of Bristol Faculty of Medicine and Dentistry Research Ethics Committee.

\section{Measurements}

Baseline data were collected between May and July 2012. Participants were asked to complete questionnaires giving basic personal data including age, sex, ethnicity, household income, educational attainment and job characteristics (Table 1). Physical activity was measured objectively using accelerometers (Actigraph GT3X+; ActiGraph LLC, FL, USA) worn on a belt around the waist during waking hours for seven days and removed for swimming and bathing. Accelerometers were set to record data at $30 \mathrm{~Hz}$. Participants also wore a personal Global Position System (GPS) receiver (QStarz BT1000XT) on the same belt during their commute to allow the journey to be spatially described. GPS data were recorded at 10-second intervals and the "assisted GPS" mode was used to enhance the precision of the GPS location data. Participants also recorded details (mode and duration) of each journey to and from work in a travel diary.

Participants were given brief instructions in the workplace about how to use the equipment by a member of the research team when the data collection equipment was distributed. Brief written instructions were also supplied, with the contact details of the research team in case of any problems or queries. All participants who returned questionnaires, travel diaries, accelerometers and/or GPS data were given a $£ 10$ gift voucher (approximately $€ 12$ or $\$ 16$ ) to acknowledge their contribution to the study.

\section{Data reduction}

Raw accelerometer data were downloaded using Actilife 6 software (ActiGraph LLC) and reintegrated to tensecond epochs for analysis and matching with GPS data. Reintegrated accelerometer data were processed using Kinesoft (v3.3.62; KineSoft, Saskatchewan, Canada) data
Table 1 Demographic characteristics of participants by mode of travel to work

\begin{tabular}{|c|c|c|c|}
\hline & $\begin{array}{c}\text { Walk } \\
(n=70)\end{array}$ & $\begin{array}{c}\text { Car } \\
(n=33)\end{array}$ & $\begin{array}{c}\text { All } \\
(n=103)\end{array}$ \\
\hline Age (years) & $36.8 \pm 12.0$ & $35.4 \pm 11.1$ & $36.3 \pm 11.7$ \\
\hline \multicolumn{4}{|l|}{ Sex (\%) } \\
\hline Male & 40.0 & 48.5 & 42.7 \\
\hline Female & 60.0 & 51.5 & 57.3 \\
\hline \multicolumn{4}{|l|}{ Ethnicity (\%) } \\
\hline White & 92.8 & 96.9 & 95.1 \\
\hline \multicolumn{4}{|l|}{ Household income (\%) } \\
\hline$<£ 10,000$ & 4.4 & 0 & 3.0 \\
\hline$£ 10,001-£ 20,000$ & 14.7 & 6.5 & 12.1 \\
\hline$£ 20,001-£ 30,000$ & 20.6 & 16.1 & 19.2 \\
\hline$£ 30,001-£ 40,000$ & 13.2 & 12.9 & 13.1 \\
\hline$£ 40,001-£ 50,000$ & 16.2 & 6.5 & 13.1 \\
\hline$>£ 50,000$ & 25.0 & 41.9 & 30.3 \\
\hline Not disclosed & 5.9 & 16.1 & 9.1 \\
\hline \multicolumn{4}{|l|}{ Education (\%) } \\
\hline No formal education & 1.4 & 3.1 & 2.0 \\
\hline $\begin{array}{l}\text { GCSE grades A-C, GCE "O" level, } \\
\text { CSE grade 1, NVQ2 or equivalent }\end{array}$ & 7.2 & 12.5 & 8.9 \\
\hline $\begin{array}{l}\text { BETC (national), BEC (national) } \\
\text { TEC (national), ONC, } \\
\text { OND or equivalent }\end{array}$ & 5.8 & 3.1 & 5.0 \\
\hline $\begin{array}{l}\text { GCE "A" level. NVQ3, Scottish } \\
\text { higher or equivalent }\end{array}$ & 11.6 & 15.6 & 12.9 \\
\hline $\begin{array}{l}\text { BETC (higher, TEC (higher), HNC, } \\
\text { HND or equivalent }\end{array}$ & 1.4 & 3.1 & 2.0 \\
\hline Degree, NVQ4 or equivalent & 49.3 & 34.4 & 44.6 \\
\hline $\begin{array}{l}\text { PhD, Masters, NVQ level } 5 \\
\text { or equivalent }\end{array}$ & 23.2 & 28.1 & 24.8 \\
\hline \multicolumn{4}{|l|}{ Occupation (\%) } \\
\hline Sedentary & 80.9 & 78.8 & 80.2 \\
\hline Standing & 14.7 & 18.2 & 15.8 \\
\hline Manual & 2.9 & 3.0 & 3.0 \\
\hline Heavy manual & 1.5 & 0 & 1.0 \\
\hline \multicolumn{4}{|l|}{ Employment pattern (\%) } \\
\hline Full time & 90.8 & 80.6 & 87.5 \\
\hline Part time & 9.2 & 19.4 & 12.5 \\
\hline
\end{tabular}

reduction software to generate outcome variables. Continuous periods of 20 minutes or more of zero values were considered to be "non-wear" time and removed. Outcome variables were total physical activity volume (mean daily accelerometer counts per minute (cpm)), moderate to vigorous physical activity (MVPA) and sedentary time, defined using validated thresholds (MVPA >1952 cpm; sedentary $<100 \mathrm{cpm}$ ) [39]. 
Participants were required to provide at least 600 minutes of accelerometer data for a single day to be considered valid, and all valid days were included in analyses. Accelerometer and GPS data were combined (accGPS) based upon the timestamp of the Actigraph data. For measurement of the journeys to and from work, the participant's workplace and home were geocoded using the full postcode, and imported into a Geographical Information System (ArcMap v10). The merged accGPS files were then imported into ArcMap and journeys to and from work visually identified and segmented from other accGPS data using the "identify" tool. Journeys were identified as a continuous (or nearcontinuous) sequence of GPS locations between the participant's home and workplace, and thus included trips to other destinations (e.g. supermarkets) if taken as part of the journey to or from work.

\section{Data analysis}

Travel diaries were used to categorise participants by their "usual" mode of travel to work over the measurement week. Only days where participants reported using the same mode of transport both to and from work were included in analyses, and participants were categorised according to the most commonly used mode of transportation. Of the 147 participants who lived within two miles $(3.2 \mathrm{~km})$ of their workplace, 23 did not provide diary data, with the remainder providing 511 weekdays of travel data comprising: walk (244 days), car (102 days), cycle (72 days) and other/mixed (93 days). Participants were categorised as "usual walkers" $(\mathrm{n}=68)$, "usual drivers" ( $n=29)$, "usual cyclists" ( $n=18)$ or "mixed/other" $(n=9)$. Participants who cycled to work were excluded from further analyses due to the inability of waist worn accelerometers to accurately record physical activity during cycling, as were data from participants using other/mixed modes of travel. Where a travel diary was not completed, usual travel mode was determined from the baseline behavioural questionnaire where possible (walk $n=6$; car $n=4$ ). The sample for analysis comprised 74 participants who usually walked to work, and 33 who usually commuted by car. Four of these participants did not provide any valid accelerometer data, and were excluded from analyses.

Analyses were confined to data recorded between 6.00 am and midnight. Mean (SD) values were computed for continuous variables and normal distribution confirmed. Differences in physical activity between travel modes (walk/car) were analysed by one-way ANOVA. Paired samples t-tests were used to compare weekday and weekend values for total physical activity (cpm), MVPA and time spent sedentary, and to investigate differences in the volume of MVPA accumulated between overall accelerometer data and spatially segmented trips. Linear regression was used to explore the association between travel mode (walk/car) and total weekday physical activity (cpm) and MVPA (minutes per day). Models were adjusted for possible confounders (age, sex, education (educated to degree level or not), income (salary below or above $£ 30,000$ per year (representing below and above mean UK household income)), work status (full/part time), occupational activity (sedentary/active)), and accelerometer wear time. Finally, one way ANOVA was used to compare total physical activity on all walking days with all car travel days.

\section{Results}

The characteristics of workplaces recruited to the study are outlined in Table 2. There was a mix of small $(\mathrm{n}=8$, $47 \%)$, medium $(\mathrm{n}=5,29 \%)$ and large $(\mathrm{n}=4,24 \%)$ workplaces in predominantly urban areas although some suburban workplaces were included $(n=4,24 \%)$. Just over half were office-based businesses $(n=9,53 \%)$ but manufacturing, transport, catering and educational establishments were also included.

The final sample comprised 103 adults (mean age $36.3 \pm$ $11.7 \mathrm{yrs} ; 57.3 \%$ female) of whom $70(50.3 \%)$ were categorised as walkers and 33 (22.4\%) as car users. Participants were predominantly white, well educated and employed in sedentary (desk-based) occupations (Table 1).

There were no statistically significant differences in any demographic characteristic between the groups. Participants included in the final sample who completed the travel diary $(\mathrm{n}=94)$ recorded 236 return journeys to work

\section{Table 2 Participating workplaces by size, type of business, location}

\begin{tabular}{llll}
\hline Workplace & Size $^{\mathbf{1}}$ & Type of Business $^{\mathbf{2}}$ & Location \\
\hline 11 & Small & Professional, scientific \& technical & City centre \\
12 & Small & Manufacturing & City centre \\
13 & Small & Professional, scientific \& technical & City centre \\
14 & Small & Professional, scientific \& technical & City centre \\
15 & Small & Professional, scientific \& technical & City centre \\
20 & Small & Professional, scientific \& technical & Suburban \\
21 & Small & Transportation & Suburban \\
22 & Small & Professional, scientific \& technical & City centre \\
17 & Medium & Education & Suburban \\
18 & Medium & Professional, scientific \& technical & City centre \\
19 & Medium & Accommodation \& food services & City centre \\
23 & Medium & Manufacturing & City centre \\
24 & Medium & Education & City centre \\
25 & Large & Public administration & City centre \\
26 & Large & Manufacturing & City centre \\
\hline 27 & Large & Financial \& insurance activities & City centre \\
\hline
\end{tabular}

${ }^{1}$ Size: Small <50; Medium 51-250; Large >250.

${ }^{2}$ Office of National Statistics, UK Standard Industrial Classification of Economic activities 2007 (SIC 2007) [40]. 
by foot, 95 by car, eight by bicycle and 56 using mixed modes. There was no record for 77 journeys. Mean selfreported journey time to work (single trip) was $19.7 \pm$ 8.3 minutes by foot and $10.7 \pm 7.6$ minutes by car.

Participants wore the accelerometer for a mean of $721.6 \pm 155.2$ minutes each day, with no difference between travel modes or sexes. Physical activity did not differ between males and females $(497.9 \pm 206.0$ versus $455.0 \pm 150.9 \mathrm{cpm}$ respectively; $\mathrm{p}=0.224$ ) and thus the sexes were analysed together. When analysed by main travel mode (Table 3), participants who walked to work had higher levels of overall weekday physical activity compared with those who travelled by car and also recorded more minutes of MVPA, but there was little difference in sedentary time.

At the weekend, total physical activity and MVPA were substantially lower in those who walked to work during the week compared to weekday values, but the physical activity of car users was essentially unchanged. There was no difference in weekend physical activity between the two travel groups. In linear regression models walking to work was associated with higher overall weekday physical activity and MVPA, contributing to 19 minutes of additional MVPA each day in adjusted models, but not sedentary time (Table 4).

Mean hourly physical activity was plotted to identify when differences in physical activity occurred during the day. Figure 1 shows that the main differences between car users and walkers occurred in the morning and late afternoon, potentially when commuting to or from work. There was no difference in physical activity between the travel groups during the main working hours ( 9 am to $4 \mathrm{pm}$ ) (walk: $347.2 \pm 187.4 \mathrm{cpm}$ vs car: $318.7 \pm 194.4 \mathrm{cpm}$; $\mathrm{p}=0.480$ ). To further explore the level of physical activity associated with walking to work, all days where the journey was conducted by foot were compared with all days where the journey was by car. Average physical activity on walking days was substantially higher than car days $(583.1 \pm 182.4$ vs $319.7 \pm 148.5 \mathrm{cpm} ; \mathrm{p}<0.001)$, with values similar to those in the analysis by individual shown in Table 3.
To explore the contribution of walking to work to total physical activity, accGPS traces recorded between $6.00 \mathrm{~mm}$ and $10.00 \mathrm{am}$, and between $4.00 \mathrm{pm}$ and $8.00 \mathrm{pm}$, were examined. Of the 74 participants who walked to work, 58 recorded GPS data for at least one journey. Overall, 321 journeys (182 to work, and 139 home from work) were recorded. Participants spent almost 22 minutes walking to work and 29 minutes walking home $(21.9 \pm 7.8$ vs $28.6 \pm 18.5$ minutes), reflecting longer routes taken to home in order to visit shops. These visits were considered to be part of the journey. Average physical activity was high during both journeys (to/from work: $4260.7 \pm 943.5$ vs $3806.3 \pm 915.8 \mathrm{cpm}$ ), though less on the journey home due to visits to shops. However, the minutes of MVPA on both the journey to and from work were similar (19.8 \pm 7.1 vs $21.0 \pm 8.9$ minutes of MVPA) since time spent in shops was not MVPA. Comparison of total MVPA (6.00 am to midnight) with MVPA recorded during the journey for the 58 participants providing any GPS data showed that the walk to and from work contributed $47.3 \%$ of participants total daily MVPA (38.0 of 80.3 minutes).

\section{Discussion}

This study explored the potential contribution of walking to work to daily physical activity in adults. In particular, we compared the baseline physical activity data from participants in a larger study to examine differences between walkers and car users. We found that activity levels were $44 \%$ higher in participants who walked to work than those travelling by car, and accumulated 57\% more MVPA. No differences were seen in physical activity during working hours or at weekends between walkers and car users. Hourly activity patterning showed that the difference between walkers and car users in weekday physical activity predominantly occurred during commuting hours, and spatial segmentation showed that the journey to and from work was responsible for the majority of the difference in weekday physical activity between those who walked to work and those who travelled by car.

An important strength of this study is the combined use of accelerometry and GPS to measure the journey to

Table 3 Weekday and weekend physical activity by usual travel mode to work on weekdays (mean \pm standard deviation)

\begin{tabular}{|c|c|c|c|c|}
\hline & All & Walk & Car & $\mathrm{p}$ \\
\hline Weekday & $(n=103)$ & $(n=70)$ & $(n=33)$ & \\
\hline Overall daily physical activity (accelerometer counts per minute (cpm)) & $473.3 \pm 176.9$ & $524.6 \pm 170.4$ & $364.6 \pm 138.4$ & $<0.001$ \\
\hline Moderate to vigorous physical activity (MVPA; minutes/day) & $69.0 \pm 28.2$ & $78.1 \pm 24.9$ & $49.8 \pm 25.2$ & $<0.001$ \\
\hline Sedentary time (minutes/day) & $586.8 \pm 71.9$ & $581.0 \pm 76.3$ & $599.2 \pm 60.5$ & 0.231 \\
\hline Weekend & $(n=68)$ & $(n=46)$ & $(n=22)$ & \\
\hline Accelerometer counts per minute & $413.2 \pm 195.9$ & $426.7 \pm 211.7$ & $385.1 \pm 158.6$ & 0.417 \\
\hline Moderate to vigorous physical activity (MVPA; minutes/day) & $53.1 \pm 30.2$ & $54.5 \pm 31.6$ & $50.2 \pm 27.7$ & 0.590 \\
\hline Sedentary time (minutes/day) & $517.4 \pm 106.0$ & $521.4 \pm 113.6$ & $509.0 \pm 90.0$ & 0.657 \\
\hline
\end{tabular}


Table 4 Linear regression analysis of total weekday physical activity, MVPA and sedentary time with travel mode

\begin{tabular}{|c|c|c|c|c|c|c|}
\hline & \multicolumn{2}{|c|}{ Total physical activity } & \multicolumn{2}{|l|}{ MVPA } & \multicolumn{2}{|l|}{ Sedentary time } \\
\hline & $\beta(95 \% \mathrm{Cl})$ & $\mathbf{p}$ & $\beta(95 \% \mathrm{Cl})$ & p & $\beta(95 \% \mathrm{Cl})$ & $\mathbf{p}$ \\
\hline Sex (male (reference)/female) & $-25.4(-104.3,53.6)$ & 0.524 & $1.8(-10.1,13.7)$ & 0.766 & $0.3(-29.6,30.2)$ & 0.984 \\
\hline Age (years) & $-3.0(-6.3,0.3)$ & 0.077 & $-0.3(-0.8,0.2)$ & 0.198 & $1.0(-0.3,2.2)$ & 0.119 \\
\hline Education (no university degree (reference)/degree) & $43.8(-41.8,129.4)$ & 0.311 & $8.6(-4.3,21.5)$ & 0.188 & $3.0(-29.4,35.4)$ & 0.854 \\
\hline Income $(\leq £ 30,000$ per annum (reference)/ $>£ 30,000)$ & $-47.2(-127.2,32.7)$ & 0.243 & $-6.7(-18.8,5.3)$ & 0.268 & $44.3(14.0,74.6)$ & 0.005 \\
\hline Occupational activity (sedentary (reference)/non-sedentary) & $-41.5(-138.0,55.0)$ & 0.394 & $-15.6(-30.1,-1.1)$ & 0.036 & $-39.6(-76.2,-3.1)$ & 0.034 \\
\hline Work status (part time (reference)/full time) & $53.4(-66.0,172.7)$ & 0.376 & $15.1(-2.9,33.0)$ & 0.099 & $10.5(-34.7,55.7)$ & 0.646 \\
\hline Accelerometer wear time (minutes per day) & $0.13(-0.15,0.40)$ & 0.364 & $0.06(0.02,0.10)$ & 0.008 & $0.17(0.07,0.28)$ & 0.002 \\
\hline Travel mode (car (reference)/walk) & $127.3(43.9,210.8)$ & 0.003 & $19.0(6.4,31.6)$ & 0.004 & $-15.6(-47.2,16.0)$ & 0.327 \\
\hline
\end{tabular}

Regression results are presented as unstandardised coefficients ( $95 \% \mathrm{Cl})$. Total weekday physical activity is accelerometer counts per minute (cpm), MVPA and Sedentary time are minutes.

work. Whilst accelerometers are commonly used to measure physical activity and can provide highly time resolved data, they are unable to record the context of physical activity. Consequently estimates of physical activity based upon, for example, hourly mean physical activity (as illustrated in Figure 1) may also include other physical activities taking place around the journey (for example walking a dog before walking to work). Combining accelerometer data with positional data from GPS receivers allowed both the level and location of physical activity to be described, and permitted identification of activity levels specifically during journeys or in particular places. These data showed that where participants took longer routes home (for example, visiting shops en route) this did not necessarily contribute to daily MVPA. Thus judging the contribution of journeys to MVPA based upon duration may be prone to error.

The study took place in a range of small, medium and large workplaces that engaged in different types of activities. As such, the study aimed to address a gap in the current research literature: the range of settings covered has been very limited and, in particular, evidence is lacking about small and medium-sized enterprises [37]. However, there are also a number of limitations to this study. The data were collected as part of a feasibility study for which we recruited a relatively small sample of predominantly well-educated younger adults, limiting the generalisability of the findings. Larger, more representative studies using objective methods are needed. The results also show high levels of MVPA, which may partly be a reflection of the accelerometer threshold used (although the threshold used is commonly applied in many studies) but also of the demographic profile of the participants. Nevertheless, the potential contribution to physical activity levels of walking the daily commute is clearly illustrated.

To our knowledge, ours is the first study in adults to use accelerometers and GPS spatial segmentation to quantify the contribution of walking to work, and our

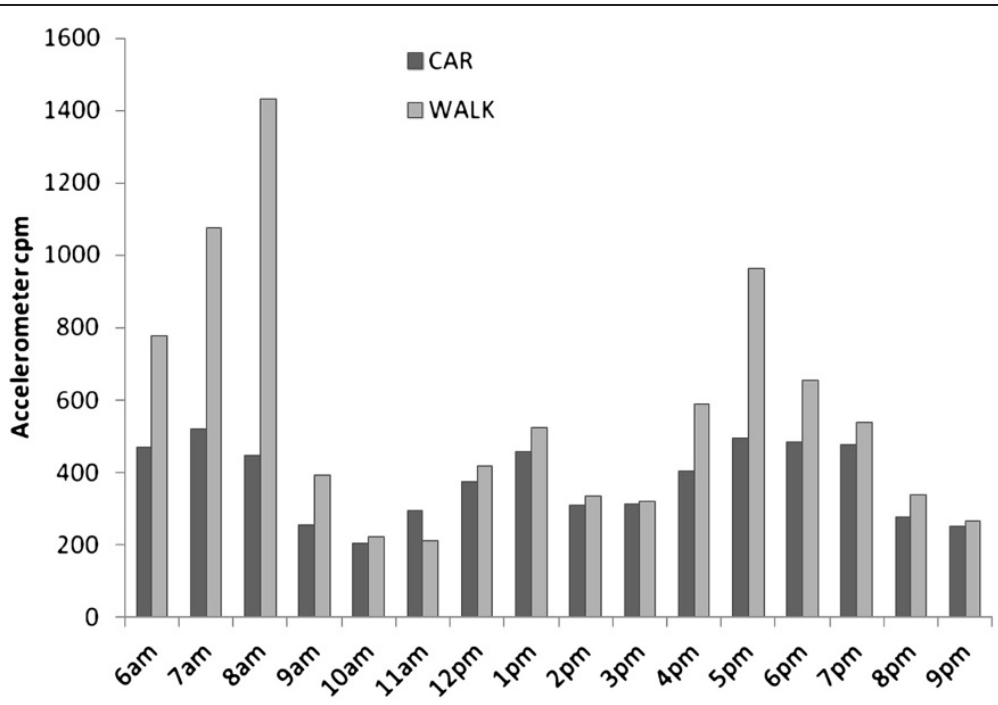

Figure 1 Mean hourly physical activity by mode of travel to/from work on weekdays. 
findings are consistent with those in children, demonstrating the substantial contribution that walking to work can make to daily physical activity. These data provide persuasive evidence to underpin interventions to increase active commuting in adults.

Public Health England recently announced several 'high-level enduring priorities' guiding their work, two of which are relevant to this study: helping people to live longer and more healthy lives by reducing preventable deaths, and; improving health in the workplace by encouraging employers to support their staff, and those moving into and out of the workforce, to lead healthier lives [41]. Similar aims are shared by governments and health practitioners throughout the world. The data presented here suggest that encouraging employees to walk to work has the potential to make a contribution to addressing these priorities.

\section{Competing interests}

The authors declare that they have no competing interests.

\section{Authors' contributions}

SA conceived and designed the study and wrote the first draft of the paper. AC analysed the accelerometer and GPS data and helped to draft the manuscript. SP coordinated the data collection and helped to draft the manuscript. All authors read and approved the final document.

\section{Acknowledgements}

We would like to thank all the participants in the Walk to Work study. This project was funded by the National Institute for Health Research Public Health Research (NIHR PHR) Programme (project number 10/3001/04). The views and opinions expressed therein are those of the authors and do not necessarily reflect those of the NIHR PHR Programme or the Department of Health. The work was undertaken with the support of The Centre for the Development and Evaluation of Complex Interventions for Public Health Improvement (DECIPHer), a UKCRC Public Health Research Centre of Excellence. Funding from the British Heart Foundation, Cancer Research UK, Economic and Social Research Council (RES-590-28-0005), Medical Research Council, the Welsh Government and the Wellcome Trust (WT087640MA), under the auspices of the UK Clinical Research Collaboration, is gratefully acknowledged. AC was supported by the NIHR Bristol Biomedical Research Unit in Nutrition, Diet and Lifestyle based at University Hospitals Bristol NHS Foundation Trust and the University of Bristol.

\section{Author details}

${ }^{1}$ School of Social and Community Medicine, University of Bristol, Canynge Hall, Whatley Road, Bristol BS8 2PS, UK. ${ }^{2}$ Centre for Exercise Nutrition and Health Sciences, School for Policy Studies, University of Bristol, Bristol BS8 $1 T Z$, UK. ${ }^{3}$ National Institute for Health Research Biomedical Research Unit in Nutrition, Diet and Lifestyle, University Hospitals Bristol NHS Foundation Trust and the University of Bristol, Bristol, UK.

Received: 13 August 2013 Accepted: 17 February 2014 Published: 11 March 2014

\section{References}

1. Department of Health: Start Active, Stay Active: A Report on Physical Activity for Health from the Four Home countries' Chief Medical Officers. London: Department of Health; 2011.

2. Warburton D, Nicol C, Bredin S: Health benefits of physical activity: the evidence. CMAJ 2006, 174(6). doi:10.1503/cmaj.051351.

3. Department of Health: UK Physical Activity Guidelines. London: Department of Health; 2011.

4. Haskell WL, Lee I-M, Pate RR, Powell KE, Blair SN, Franklin BA, Macera CA, Heath GW, Thompson PD, Bauman A: Physical activity and public health: updated recommendation for adults from the American College of
Sports Medicine and the American Heart Association. Med Sci Sports Exerc 2007, 39(8):1423-1434.

5. The Information Centre: Health Survey for England 2008: Volume 1 Physical Activity and Fitness. London: The information Centre for health and social care; 2010

6. Rutherford L, Sharp C, Bromley CN (Eds): The Scottish Health Survey 2011. Edinburgh: Scottish Executive Health Department; 2012.

7. Department of Health: Be Active, be Healthy: A Plan for Getting the Nation Moving. London: Department of Health; 2009.

8. Physical Activity Task Force: Let's Make Scotland More Active: A Strategy for Physical Activity. Edinburgh: Scottish Executive; 2003.

9. Department of Health: Healthy Lives, Healthy People: Our Strategy for Public Health in England. London: Department of Health; 2010.

10. Bassett D, Pucher J, Buehler R, Thompson D, Crouter S: Walking, cycling, and obesity rates in Europe, North America and Australia. J Phys Act Health 2008, 5:795-814.

11. World Health Organisation: Economic Assessment of Transport Infrastructure and Policies: Methodological Guidance on the Economic Appraisal of Health Effects Related to Walking and Cycling. Denmark: World Health Organization; 2007.

12. Saunders L, Green J, Petticrew M, Steinbach $R$, Roberts $H$ : What are the health benefits of active travel? A systematic review of trials and cohort studies. PLoS One 2013, 8(8):e69912. doi:10.1371/journal.pone.0069912.

13. Laverty A, Mindell J, Webb E, Millett C: Active travel to work and cardiovascular risk factors in the United Kingdom. Am J Prev Med 2013, 45(3):282-288.

14. Hamer M, Chida Y: Active commuting and cardiovascular risk: a metaanalytic review. Prev Med 2008, 46:9-13.

15. Bopp M, Kaczynski A, Campbell M: Health-related factors associated with mode of travel to work. Int J Environ Res Publ Health 2013. Article ID 242383. doi.org/10.1155/2013/242383.

16. Davis A: Value for Money: An Economic Assessment of Investment in Walking and Cycling. London: Department of Health and Government Office of the South West; 2010

17. Ogilvie D, Foster CE, Rothrie H, Call N, Hamilton V, Fitzsimons CF, Mutrie N: Interventions to promote walking systematic review. BMJ 2007. doi:10.1136/bmj.39198.722720.BE.

18. Panter J, Desousa C, Ogilvie D: Incorporating walking or cycling into car journeys to and from work: the role of individual, workplace and environmental characteristics. Prev Med 2013, 56:211-217.

19. de Nazelle A, Nieuwenhuijsen MJ, Anto JM, Brauer M, Briggs D, BraunFahrlander C, Cavill N, Cooper AR, Desqueyroux H, Fruin S, Hoek G, Panis LI, Janssen $N$, Jerrett $M$, Joffe M, Andersen ZJ, van Kempen E, Kingham S, Kubesch N, Leyden KM, Marshall JD, Matamala J, Mellios G, Mendez M, Nassif H, Ogilvie D, Peiro R, Perez K, Rabl A, Ragetti M, et al: Improving health through policies that promote active travel: A review of evidence to support integrated health impact assessment. Environ Int 2011, 37:766-777.

20. Jarrett J, Woodcock J, Griffiths UK, Chalabi Z, Edwards P, Roberts I, Haines A: Effect of increasing active travel in urban England and Wales on costs to the National Health Service. Lancet 2012, 379:2198-2205.

21. Morris J, Hardman A: Walking to health. Sports Med 1997, 23:306-332.

22. Ainsworth B, Haskell WL, Whitt MC, Irwin ML, Swartz AM, Strath SJ, O'Brien WL, Bassett DR, Schmitz KH, Emplaincourt P, Jacobs DR, Leon AS: Compendium of physical activities: an update of activity codes and MET intensities. Med Sci Sports Exerc 2000, 32(Supp I):S498-S516.

23. Scottish Executive: A Walking Strategy for Scotland: Consultation Document Edinburgh: Scottish Executive Development Department; 2003.

24. Department for Transport: Walking and Cycling: An Action Plan. London: Department of Transport; 2004.

25. Welsh Assembly Government: A Walking and Cycling Action Plan for Wales. Wales, UK: Welsh Assembly; 2008.

26. Coote A: What health services could do about climate change. BMJ 2006, 332:1343-1344.

27. Department for Transport: National Travel Survey Statistical Release. London: Department of Transport; 2012.

28. Mutrie N, Carney C, Blamey A, Crawford F, Aitchison T, Whitelaw A: "Walk in to Work Out": a randomised controlled trial of a self help intervention to promote active commuting. J Epidemio/ Community Health 2002, 56:407-412

29. Sahlqvist S, Song Y, Ogilvie D: Is active travel associated with greater physical activity? The contribution of commuting and non-commuting 
active travel to total physical activity in adults. Prev Med 2012

55:206-211.

30. Prince SA, Adamo KB, Hamel ME, Hardt J, Connor Gorber S, Tremblay M: A comparison of direct versus self-report measures for assessing physical activity in adults: a systematic review. Int J Behav Nutr Phys Act 2008, 5:56. doi:10.1186/1479-5868-5-56.

31. Vuillemin A, Rostami C, Maes L, Van Cauwenberghe E, Van Lenthe FJ, Brug J, De Bourdeaudhuij I, Oppert JM: Worksite physical activity interventions and obesity: a review or Euopean studies (the HOPE Project). Obes Facts 2011, 4:479-488.

32. Gordon-Larsen P, Boone-Heinonen J, Sidney S, Sternfeld B, Jacobs DR Jr, Lewis CE: Active commuting and cardiovascular disease risk the CARDIA study. Arch Intern Med 2009, 169(13):1216-1223.

33. Faulkner GE, Buliung RN, Flora PK, Fusco C: Active school transport, physical activity levels and body weight of children and youth: a systematic review. Prev Med 2009, 48(Issue 1):3-8.

34. Cooper AR, Jago R, Southward EF, Page AS: Active travel and physical activity across the school transition: the PEACH project. Med Sci Sports Exerc 2012, 44(10):1890-1897.

35. Smith L, Sahlqvist S, Ogilvie D, Jone A, Corder K, Griffin S, van Sluijs E: Is a change in mode of travel to school associated with a change in overall physical activity levels in children? Longitudinal results from the SPEEDY study. Int J Behav Nutr Phys Act 2012, 9:134. doi:10.1186/1479-5868-9-134.

36. Southward E, Page A, Wheeler B, Cooper A: Contribution of the school journey to daily physical activity in children aged 11-12 years. Am J Prev Med 2012, 43(2):201-204.

37. National Institute for Health and Clinical Evidence: Promoting Physical Activity in the Workplace. NICE Public Health Guidance 13. London: Department of Health; 2008.

38. National Institute for Health Research. Public Health Research programme: Employer schemes to encourage walking to work: feasibility study incorporating an exploratory randomised controlled trial. [http://www. phr.nihr.ac.uk/funded_projects/10_3001_04.asp]

39. Freedson PS, Melanson E, Sirard J: Calibration of the computer science and applications, inc. accelerometer. Med Sci Sports Exerc 1998, 30(5):777-781.

40. Office of National Statistics: UK Standard Industrial Classification of Economic activities 2007 (SIC 2007). Basingstoke, UK: Office of National Statistics; 2007.

41. Public Health England: Our Priorities for 2013/14. London: Public Health England; 2013.

doi:10.1186/1479-5868-11-37

Cite this article as: Audrey et al:: The contribution of walking to work to adult physical activity levels: a cross sectional study. International Journal of Behavioral Nutrition and Physical Activity 2014 11:37.

\section{Submit your next manuscript to BioMed Central and take full advantage of:}

- Convenient online submission

- Thorough peer review

- No space constraints or color figure charges

- Immediate publication on acceptance

- Inclusion in PubMed, CAS, Scopus and Google Scholar

- Research which is freely available for redistribution

Submit your manuscript at www.biomedcentral.com/submit
() Biomed Central 